Trauma Berufskrankh 2010 · 12 [Suppl 1]:75-77 DOI 10.1007/s10039-009-1559-1

Online publiziert: 1. November 2009

๑) Springer Medizin Verlag 2009

A.O. Scholz · E. Muwazi $\cdot$ W. Hasse $\cdot$ H.-R. Kortmann

Klinik für Unfallchirurgie und Orthopädie, BG-Unfallklinik Duisburg GmbH

\title{
Blutgruppe Bombay
}

tungen. Weltweit sind etwa 20.000 Träger des Bombay-Phänotyps bekannt.

Problematisch ist sowohl beim Bombayals auch beim Para-Bombay-Phänotyp die Bildung von Anti-H-Antikörpern. Diese richten sich gegen die heterogenetische Grundsubstanz „H“, welche bei den Blutgruppen $\mathrm{O}, \mathrm{A}, \mathrm{B}$ und $\mathrm{AB}$ vorhanden ist. Somit kommt es bei Personen vom Typ Bombay mit einem Anti-H-Antikörper auch zu einer Antigen-Antikörper-Reaktion mit Erythrozyten der Blutgruppe o. Dies ergibt in Konsequenz, dass bei diesen Personen nur Blutkonserven der Untergruppe Bombay transfundiert werden können.

Wir berichten im Folgenden über einen jungen Patienten mit einem ausgedehnten Weichteiltrauma des Unterschenkels und einer transfusionspflichtigen Anämie bei zufällig festgestelltem Bombay-Phänotyp.

Das H-Antigen ist ein wichtiges Antigen zur Vorstufe der Biosynthese der Aund B-determinanten Strukturen [5]. Im kodierenden Gen finden sich neben dem aktiven $\mathrm{H}$-Allel, welches die Bildung der H-Substanz kontrolliert, auch eine Reihe von inaktiven h-Allelen, die für das Auftreten der seltenen Bombay- und Para-Bombay-Phänotypen verantwortlich sind. Kommt es zu einer genetischen Fehlausprägung im für das H-Antigen kodierenden Gen, entstehen so genannte H-defiziente Varianten (Genotyp hh). Nach der heutigen Definition handelt es sich dabei

- entweder um den so genannten Bombay-Phänotyp als H-defiziente Nichtsekretorvariante

- oder den Para-Bombay-Phänotyp mit $\mathrm{H}$-defizientem Sekretorverhalten.

Beide Untergruppen zeigen keinerlei ABH-Aktivität an den Erythrozyten. Im Gegensatz zum Bombay-Phänotyp finden sich jedoch im Para-Bombay-Phänotyp noch $\mathrm{ABH}$-Antigene in den übrigen Körpergeweben.

Entdeckt wurde der Bombay-Phänotyp im Jahre 1952 von Bhende et al. [2] in Bombay/Indien. Seine Inzidenz in Indien wird auf 1:760o beziffert. Bevorzugt hierbei ist der Süden um die Region Maharashtra betroffen, wo Blutsverwandtenehen üblich sind. In anderen Ländern gibt es bisher nur kasuistische Einzelbeobach-
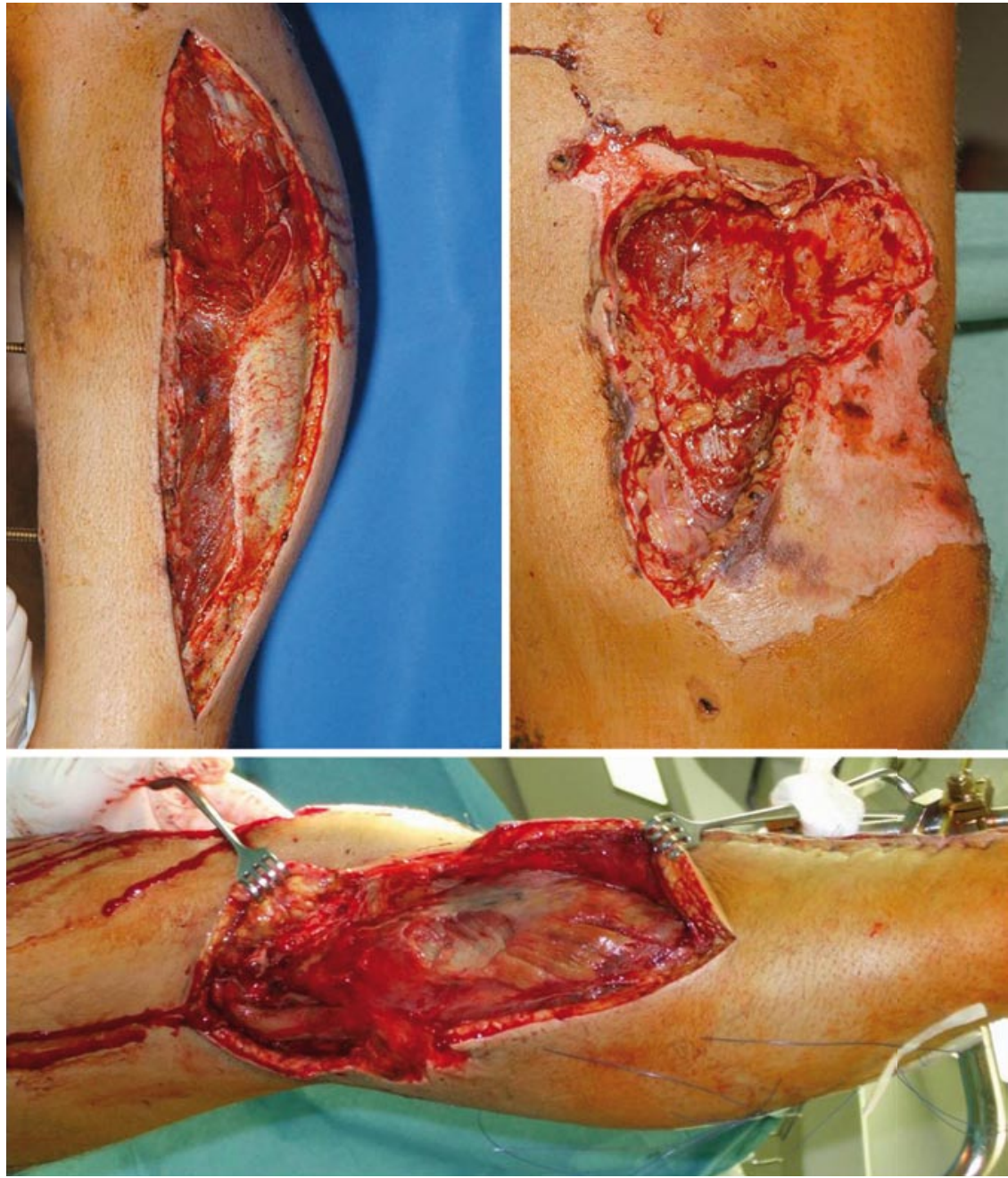

Abb. $1 \Delta$ Ausgedehnte Weichteildefekte am linken Unter- und Oberschenkel mit Kompartmentspaltung des Unterschenkels 

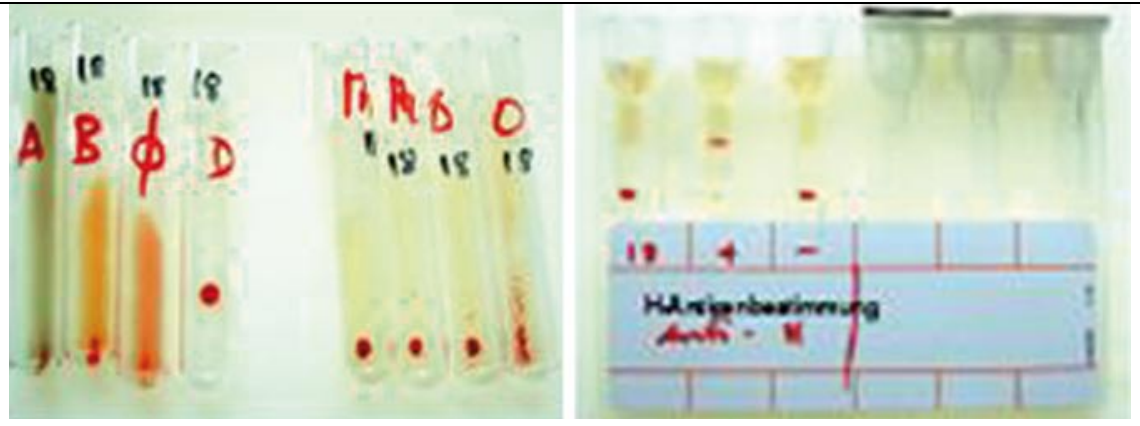

Abb. $2 \Delta$ Positiver Coombs- und Antikörpertest des Patienten

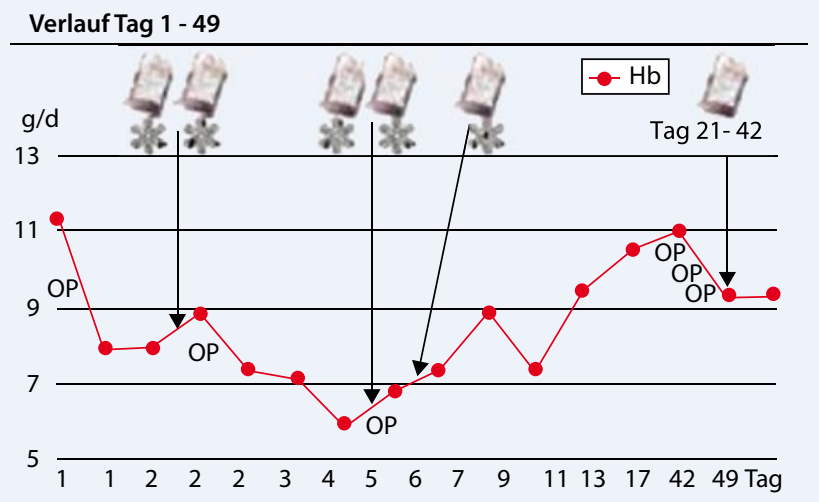

Abb. $3<$ Verlauf der Hb-Werte mit Gabe der Erythrozytenkonzentrate, Stern Kryokonservate
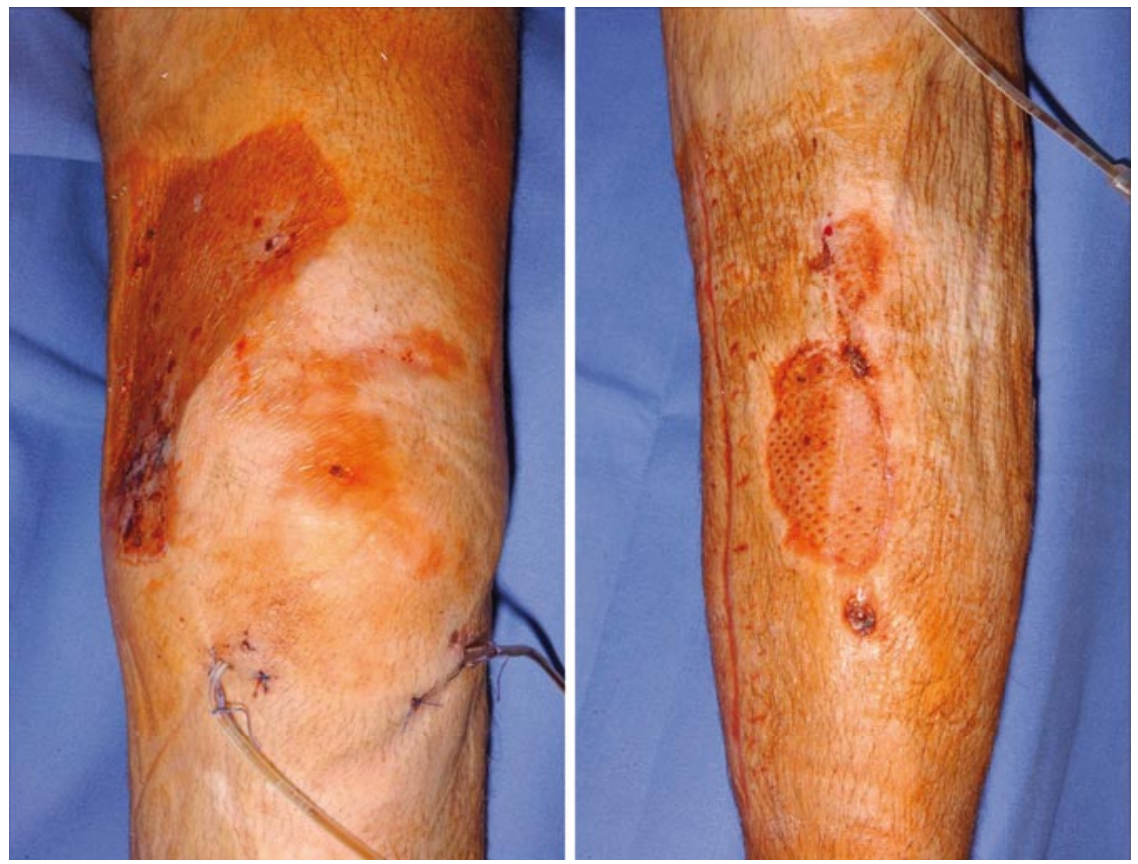

Abb. 4 \ Wunden nach Sekundärnaht und Meshgraft-Deckung

\section{Kasuistik}

Der 26-jährige Müllwerker wurde im April 2008 nach einem Überrolltrauma durch den Müllwagen in die BG-Unfallklinik Duisburg gebracht. Initial zeigte sich bei der Aufnahme eine ausgedehnte
Quetschverletzung des linken Ober- und Unterschenkels mit Kompartmentsyndrom des Beins (• Abb. 1). Ebenso fand sich ein weit reichendes subkutanes Décollement ohne knöcherne Verletzungen. Noch am Aufnahmetag wurde eine operative Revision durchgeführt und Blut zur Blutgruppenbestimmung und Anforderung von Kreuzblut abgenommen.

Im Labor wurde bei der Blutgruppentestung die Blutgruppe o mit positiver Reaktion der Blutgruppe-o-Erythrozyten in der Serumgegenprobe entdeckt (- Abb. 2). Ebenso fand sich ein positiver Antikörpersuchtest in allen Zellen.

Der Aufmerksamkeit der diensthabenden MTA (medizinisch-technische/-r Assisten/-in) ist es zu verdanken, dass bei Verdacht auf die Blutgruppe Bombay eine sofortige Rücksprache mit dem Blutspendezentrum des Deutschen Roten Kreuzes West in Breitscheid erfolgte. Noch am selben Tag wurde eine immunhämatologische Untersuchung im Zentrum für Transfusionsmedizin in Breitscheid, mit der Diagnose „Blutgruppe Bombay“ $\left(\mathrm{o}_{\mathrm{h}}\right)$, durchgeführt. Nach dieser Diagnosestellung wurden sofort durch das Blutspendezentrum Breitscheid und Hagen 2 kryokonservierte Erythrozytenkonzentrate freigegeben. Ebenso wurde unverzüglich am Folgetag die Untersuchung aller Verwandten des Patienten in die Wege geleitet. Hierbei wurde festgestellt, dass die Eltern miteinander verwandt waren. Aufgrund der nur wenigen kryokonservierten Erythrozytenkonzentrate und fehlender Bombay-Phänotypen in der Verwandtschaft wurde Kontakt mit dem „German Rare Donor-Program“ der Universität Ulm aufgenommen.

Der Patient wurde mehrfachen Operationen unterzogen. Er wurde am 2. Tag transfusionspflichtig. Hierbei wurden die ersten 2 Erythrozytenkonzentrate gegeben. Insgesamt mussten 6 Erythrozytenkonzentrate verabreicht werden, wobei es sich bei dem Letzten um ein Frischkonzentrat aus Ulm handelte. Der tiefste Hb-Wert (Hämoglobin) konnte am 4. Tag nach dem Unfall mit 5,9 g/dl gemessen werden ( $\bullet$ Abb. 3). Parallel zur Konservengabe wurden Eisen, Folsäure und Erythropoetin zur Steigerung der körpereigenen Erythrozytenproduktion verabreicht.

Im weiteren Verlauf konnten die Wunden durch Sekundärnähte und MeshgraftDeckung verschlossen werden (• Abb. 4), und der Patient wurde am 56. Tag mit einem Hb-Wert von 12,4 g/dl nach Hause entlassen. 


\section{Diskussion}

Der entscheidende Unterschied zwischen Personen mit den normalen Blutgruppen A, B und o zu einer Person mit der Blutgruppe $\mathrm{O}_{\mathrm{h}}$ (Bombay-Phänotyp) besteht darin, dass im Serum nicht nur die üblichen Anti-A- und Anti-B-Antikörper als natürliche (so genannte LandsteinerAntikörper) vorkommen, sondern regelmäßig auch Anti-H-Antikörper [5]. Diese richten sich gegen die Erythrozyten und reagieren mit diesen in Form einer akuten Erythrozytenagglutination $[4,6]$.

Die Anti-H-Antikörper bei einer Person mit Bombay-Phänotyp sind vorzugsweise Antikörper der IGM-Klasse (Immunglobulin $\mathrm{M}$ ), welche nicht plazentagängig sind und damit auch nicht zu einer Neugeborenenerythroblastose führen. $\mathrm{Zu}$ differenzieren ist dieses Anti-H von dem so genannten Kälteagglutinin-Anti- $\mathrm{H}$, welches bei niedrigen Temperaturen zu einer Erythrozytenagglutination führt.

Personen mit dem Bombay-Phänotyp dürfen ausnahmslos nur Blut eines Spenders der gleichen Blutgruppe erhalten [ $[1$, $2,3,4,5,6]$, daraus ergibt sich für Notsituationen mit vitaler Transfusionsindikation das Problem der Blutversorgung. Eine Möglichkeit, für Patienten mit der Blutgruppe $o_{h}$ Blut zu gewinnen, stellt die Spende von Eigenblut dar, welches konserviert und bei Bedarf retransfundiert werden kann. In der Akutsituation bei unbekannten Trägern des Bombay-Phänotyps muss jedoch auf kryokonservierte Erythrozytenkonzentrate zurückgegriffen werden. Diese sind über das „German Rare Donor-Program“ der Universität Ulm abzurufen. Kennzeichen der kryokonservierten Erythrozytenkonzentrate ist, dass sie bei einer Lagerungstemperatur unter $80^{\circ} \mathrm{C}$ auf flüssigem Stickstoff bis zu 10 Jahre haltbar sind. Nach dem Auftauvorgang ist jedoch eine Transfusion innerhalb von $24 \mathrm{~h}$ zwingend.

Aufgrund der nur extrem geringen Inzidenz der Phänotypträger (20.00o weltweit), ist es notwendig, bei Identifikation eines solchen diesen zur Blutspende anzuregen, um ausreichend kryokonservierte Erythrozytenkonzentrate in Bereitstellung zu haben. Ebenso sollte bei BombayTrägern der familiäre Hintergrund unter- sucht werden, um evtl. weitere BombayPhänotyp-Träger zu identifizieren.

Nebenbei bemerkt sei, dass BombayPhänotyp-Träger anscheinend reduzierte Plasmaspiegel des Von-Willebrand-Faktors (Faktor VIII: vWF) besitzen [4]. Aufgrund der dadurch gestörten Blutgerinnung kann es zu prolongierten Blutungen im Rahmen von Unfällen kommen.

In unserem Fall ist es nur der Aufmerksamkeit der MTA zu verdanken, dass die Diagnose des Bombay-Phänotyps rechtzeitig erfolgte. Bei irrtümlicher o-negativTransfusion wäre es zu einer akuten Hämolyse mit wahrscheinlich lebensbedrohlichen Problemen gekommen. Aufgrund der sofortigen Diagnose und des logistischen Aufwandes mit Transport von kryokonservierten und Frischblutkonzentraten aus den lokalen Blutspendezentren und der Universität Ulm konnte in unserem Fall ein guter Verlauf mit gutem Ausgang erzielt werden.

\section{Korrespondenzadresse}

\section{Dr. A.O. Scholz}

Klinik für Unfallchirurgie und Orthopädie, BG-Unfallklinik Duisburg GmbH, Großenbaumer Allee 250, 47249 Duisburg armin-olaf.scholz@bgu-duisburg.de

Interessenkonflikt. Der korrespondierende Autor gibt an, dass kein Interessenkonflikt besteht.

\section{Literatur}

1. Bhattacharya S, Makar Y, Laycock RA et al (2002) Outcome of consecutive pregnancies in a patient with Bombay $\left(0_{h}\right)$ blood group. Transfus Med 12:379-381

2. Bhende YM, Deshpande CK, Bhatia HM et al (1952) $\mathrm{A}_{\text {"n }}$ new" blood group character related to the $\mathrm{AB} 0$ system. Lancet 1:903-904

3. Lin-Chu M, Broadberry RE (1990) Blood transfusion in the para-Bombay phenotype. Br J Haematol 75:568-572

4. O'Donnell JS, McKinnon TAJ, Crawley TB et al (2005) Bombay phenotype is associated with reduced plasma-vWF levels and increased susceptibility to ADAMTS13 proteolysis. Blood 106(6):1988-1991

5. Schenkel-Brunner H (2001) Die biochemischen Grundlagen der Untergruppen des ABO-Systems. Wien Klin Wochenschr 113/20-21:787-798

6. Schricker KTh, Neidhardt B, Hacker R, Kail R (1983) Herzoperation bei einer Patientin mit der Blutgruppe $0_{\mathrm{h}}$ (Bombay-Phänotyp). Dtsch Med Wochenschr 108:61-63
Trauma Berufskrankh 2010 · 12 [Suppl 1]:

75-77

DOI 10.1007/s10039-009-1559-1

C) Springer Medizin Verlag 2009

\section{A.O. Scholz - E. Muwazi · W. Hasse · H.-R. Kortmann Blutgruppe Bombay}

\section{Zusammenfassung}

Das AB0-Blutgruppen-System weist zahlreiche Untergruppen auf. Durch eine Störung des Gens für die Vorläufersubstanz H kann es zur Ausbildung von Antikörpern gegen Blutgruppenmerkmale auf den Erythrozyten $(\mathrm{H}-$ Antikörper) kommen. Diese Variante nennt man Bombay-Phänotyp $\left(0_{h}\right)$. Sein Merkmal ist eine Erythrozytenagglutination bei Gabe von Erythrozyten der Gruppe 0 . Weltweit sind 20.000 Träger (höchste Inzidenz in Indien) bekannt. Bombay-Träger können nur Bombay-Blut empfangen. In unserem Fall handelte es sich um ein ausgeprägtes Weichteiltrauma bei einem unbekannten BombayTräger mit transfusionspflichtiger Anämie. Nur durch einen hohen organisatorischen und logistischen Aufwand und die Gabe von Bombay-Kryokonserven konnte die definitive Versorgung ohne Komplikationen erfolgen.

\section{Schlüsselwörter}

ABO-Blutgruppen · Anämie · Bombay-Phänotyp $\cdot \mathrm{H}$-Antigen $\cdot$ Erythrozytenagglutination

\section{The Bombay blood group type}

\section{Abstract}

The $A B 0$ blood group system exhibits numerous subgroups. By a disturbance of the gene for the $\mathrm{H}$ antigen, antibodies against blood group identifiers on the erythrocytes $(\mathrm{H}$ antibody) are produced. This variant is called the Bombay phenotype $\left(0_{h}\right)$. Characteristic of the Bombay phenotype is erythrocyte agglutination with the administration of group 0 erythrocytes. Worldwide, 20,000 carriers are known, with the highest incidence in India. Bombay carriers can receive only Bombay blood. We present the case of a previously unknown Bombay carrier who sustained pronounced soft tissue trauma in an accident. As a result of an intensive diagnostics process, the rare Bombay phenotype was detected, and the patient was able to receive appropriate blood for transfusions. Only through high organizational and logistic efforts and with the administration of Bombay blood can definitive care without complications be provided to affected individuals.

\section{Keywords}

AB0 blood groups - Anemia - Bombay phenotype $\cdot \mathrm{H}$ antigen $\cdot$ Erythrocyte agglutination 